\title{
Efavirenz: A review of the epidemiology, severity and management of neuropsychiatric side-effects
}

\author{
R Gaida, ${ }^{1}$ MPharm; I Truter, ${ }^{1,2}$ DCom, BPharm, MSc, PhD; C Grobler, ${ }^{3}$ MB ChB, DOH, FCPsych, MMed (Psych), MD \\ ${ }^{1}$ Department of Pharmacy, Nelson Mandela Metropolitan University, Port Elizabeth, South Africa \\ ${ }^{2}$ Drug Utilisation Research Unit, Nelson Mandela Metropolitan University, Port Elizabeth, South Africa \\ ${ }^{3}$ Department of Psychology, Nelson Mandela Metropolitan University; Elizabeth Donkin Hospital, Port Elizabeth, South Africa
}

\section{Corresponding author: I Truter (ilse.truter@nmmu.ac.za)}

\begin{abstract}
South Africa has the highest proportion of HIV-positive people in the world. HIV cannot be cured; however, there are several major classes of drugs used in its management. Efavirenz is one such agent of the class non-nucleoside reverse transcriptase inhibitors which inhibits the replication of the virus. Efavirenz is associated with causing neuropsychiatric side-effects (NPSEs), with almost 50\% of patients experiencing at least one NPSE while on treatment. The NPSEs tend to occur within the first few days of initiation of therapy and resolve spontaneously within the first 4 - 6 weeks, with the most commonly reported being dizziness, insomnia, headache, abnormal dreams and impaired concentration. The plasma level of efavirenz and genetic polymorphisms are thought to play a role in the development of such NPSEs. NPSEs need to be treated according to severity. If necessary, efavirenz may be replaced with nevirapine or lopinavir/ritonavir. It should be remembered that nevirapine may also produce some severe side-effects such as skin abnormalities and hepatotoxicity. The monitoring of patients receiving efavirenz therapy should be ongoing, with those with a history of mental illness requiring closer monitoring than others.
\end{abstract}

S Afr J Psychiatr 2015;21(3):94-97. DOI:10.7196/SAJP.8260

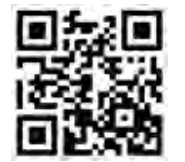

South Africa (SA) has the highest proportion of HIVpositive people in the world. In 2013, an estimated $10 \%$ of the population was HIV-positive, which amounted to 5.26 million people. ${ }^{[1]}$ For adults between the ages of 15 and 49 years, an estimated $15.90 \%$ were HIVpositive. ${ }^{[1]}$ However, SA has made positive changes in managing the HIV epidemic. The number of people on antiretroviral therapy (ART) has increased, and there have been fewer AIDS-related deaths from 2005 to 2011 . $^{[2]}$

\section{Antiretrovirals used in the management of HIV}

HIV cannot be cured; however, there are several major classes of drugs used in its management. The five classes of drugs used for the management of HIV are entry inhibitors, fusion inhibitors, integrase inhibitors, protease inhibitors and reverse transcriptase inhibitors (nucleoside reverse transcriptase inhibitors and non-nucleoside reverse transcriptase inhibitors). ${ }^{[3]}$ These agents act through various mechanisms to stop the replication of HIV. The recommended regimens for the management of HIV in SA are summarised in Table 1. ${ }^{[4]}$

Efavirenz is a non-nucleoside reverse transcriptase inhibitor that produces its antiretroviral activity by binding directly to the enzyme reverse transcriptase, thus inhibiting replication of the virus. ${ }^{[5]}$ Efavirenz possesses a long half-life of $40-55$ hours $^{[6]}$ and is predominantly metabolised in the liver by the CYP450 enzyme system. The specific isoform within the system most important for the metabolism of efavirenz is CYP2B6. ${ }^{[7-9]}$
Almost $50 \%$ of patients on efavirenz experience at least one NPSE. ${ }^{[10-12]}$ In spite of this, efavirenz is part of the first-line regimen of HIV management in SA. The NPSEs tend to occur within the first few days after initiation of therapy and then resolve spontaneously within the first 4 - 6 weeks. ${ }^{[10]}$ The most commonly reported NPSEs are dizziness, insomnia, headache, abnormal dreams and impaired concentration. ${ }^{[1,13,14]}$ An increased risk of suicidality has been a concern with efavirenz; however, there is conflicting opinion regarding this. ${ }^{[11,12,15]}$ In light of this, patients with an active psychiatric illness being considered for efavirenz therapy should be evaluated in terms of suicide risk, and these patients should be closely monitored after the initiation of therapy.

HIV infection is now regarded as a 'chronic' condition; ${ }^{[16]}$ therefore, it is important to understand the long-term effects of efavirenz. Studies ${ }^{[12,17,18]}$ have been done to assess the long-term effects and have shown that NPSEs may persist for up to 2 - 3 years following initiation of efavirenz. Dizziness, sleep disturbances, abnormal dreams and light-headedness were the persisting symptoms. ${ }^{[12,17,18]}$ The 2010 Clinical Guidelines for the Management of HIV and AIDS in Adults and Adolescents in South Africa ${ }^{[19]}$ stated that the recommended safety monitoring for the NPSEs of efavirenz is 'clinical. The term 'clinical' is left open to interpretation with no specific symptoms that should be taken into account when considering a diagnosis being listed. This indicates that it is the responsibility of the clinician to determine whether or not side-effects constitute a clinical change in the patient's condition and what management strategies need to be implemented. These Guidelines also advise that efavirenz should be 
Table 1. Standardised first-line antiretroviral therapy regimens for adults and adolescents in $\mathrm{SA}^{[4]}$

\begin{tabular}{|c|c|c|}
\hline Patient group & Regimen & Recommendations \\
\hline $\begin{array}{l}\text { Adolescents }>15 \text { years and weighing } \\
>40 \mathrm{~kg} \\
\text { Adults }\end{array}$ & $\begin{array}{l}\text { Tenofovir }+ \text { lamivudine (or emtricitabine) } \\
+ \text { efavirenz to be provided as a fixed-dose } \\
\text { combination }\end{array}$ & $\begin{array}{l}\text { Replace efavirenz with nevirapine in patients with significant } \\
\text { psychiatric comorbidity or intolerance to efavirenz and where } \\
\text { the neuropsychiatric toxicity of efavirenz may impair daily } \\
\text { functioning, e.g. shift workers }\end{array}$ \\
\hline
\end{tabular}

All hepatitis B virus co-infection

Adults and adolescents on stavudine Change stavudine to tenofovir (no patient should be on stavudine)
Switch to tenofovir if virally suppressed and the patient has normal creatinine clearance, even if stavudine is well tolerated. If the viral load is $>1000$ copies $/ \mathrm{mL}$, manage as treatment failure and consider switching to second line

If adolescent weight $<40 \mathrm{~kg}$, align with paediatric regimen Comments

\begin{tabular}{|c|c|}
\hline Contraindication & Substitution drug \\
\hline $\begin{array}{l}\text { Contraindications to efavirenz: } \\
\text { Significant psychiatric comorbidity } \\
\text { Intolerance to efavirenz } \\
\text { Impairment of daily function }\end{array}$ & $\begin{array}{l}\text { Tenofovir }+ \text { emtricitabine (or lamivudi } \\
\text { nevirapine or lopinavir/ritonavir }\end{array}$ \\
\hline $\begin{array}{l}\text { Contraindications to tenofovir: } \\
\text { Creatinine clearance of }<50 \mathrm{~mL} / \mathrm{m}\end{array}$ & $\begin{array}{l}\text { Abacavir + lamivudine + efavirenz (or } \\
\text { nevirapine) }\end{array}$ \\
\hline
\end{tabular}

If CD $4<250$ cells $/ \mathrm{mm}^{3}$ (females) and $<400$ cells $/ \mathrm{mm}^{3}$ (males), give nevirapine $200 \mathrm{mg}$ daily for 2 weeks, then $200 \mathrm{mg}$ twice daily If CD $4 \geq 250$ cells $/ \mathrm{mm}^{3}$ (females) and $\geq 400$ cells $/ \mathrm{mm}^{3}$ (males), use lopinavir/ritonavir (two tablets 12 hourly)

Renal disease or the use of other nephrotoxic drugs, e.g. aminoglycosides

Multidrug-resistant TB treatment

$\mathrm{TB}=$ tuberculosis

avoided in patients with untreated depression and patients receiving psychoactive drugs. ${ }^{[19]}$ The 2014 Guidelines $^{[4]}$ warn that efavirenz may cause persistent central nervous system toxicity such as abnormal dreams, depression or mental confusion, and that these side-effects are more likely to occur in patients with current or previous depression or other mental disorder or if the efavirenz is taken during the day. ${ }^{[4]}$ The efavirenz package insert does not state that efavirenz is contraindicated in psychiatric patients. ${ }^{[20]}$ It does, however, mention that efavirenz can cause NPSEs, although the incidence is stated as rare and includes only the following symptoms: anxiety, apathy, emotional lability, euphoria, hallucinations and depression. ${ }^{[20]}$

\section{Epidemiology of neuropsychiatric effects}

The three main risk factors for the development of NPSEs in HIVpositive patients are: pre-existing mental conditions, HIV disease progression and ART. The plasma level of efavirenz is thought to play a role in the development of NPSEs, as are genetic polymorphisms in certain population groups. ${ }^{[10,21]}$

\section{Plasma level}

The plasma level of efavirenz seems to have a place in predicting the incidence of NPSEs since the stepwise dosing of efavirenz is shown to decrease both the incidence and severity of NPSEs. ${ }^{[10]}$ However, studies $^{[22,23]}$ attempting to determine the exact plasma level above which patients are at risk of developing these NPSEs have produced inconsistent data.

\section{Genetic polymorphisms}

Efavirenz is predominantly metabolised by the CYP450 enzyme system, specifically by the CYP2B6 isoform..$^{[7-9]}$ This enzyme is highly susceptible to polymorphism and these polymorphs play a role in the variability of efavirenz plasma concentrations. ${ }^{[9]}$ Functionally deficient alleles may result in higher plasma levels of efavirenz in patients receiving a standard dose of $600 \mathrm{mg}$ daily because of decreased metabolism of efavirenz. ${ }^{[9]}$ The frequency of these deficient alleles varies among populations, but has been shown to be more common in patients of African descent. ${ }^{[9]}$ Such patients should therefore be monitored more closely as they are more likely to experience a higher plasma level of efavirenz and be susceptible to NPSEs. In an SA context, there are patients who possess the allelic variations, but there is no evidence to suggest that patients would benefit from routine genotyping and measurement of efavirenz plasma levels in terms of therapeutic outcomes. ${ }^{[2]}$ Therefore the current doses are sufficient.

\section{Comparative studies between efavirenz and other antiretrovirals}

Studies have been conducted comparing the incidence of NPSEs caused by efavirenz and other antiretroviral agents. Efavirenz has been compared with nevirapine, protease inhibitors, etravirine and raltegravir. $^{[25-27]}$ All of these studies ${ }^{[25-27]}$ analysed have shown that the virological efficacy of efavirenz is not inferior to any other regimen. Although efavirenz demonstrated a higher incidence of NPSEs in all cases, the symptoms tended to be mild and necessitated discontinuation in only small numbers of patients. However, the studies $^{[17,27]}$ noted that there were incidences of delayed onset of NPSEs associated with efavirenz, which refer to patients who develop these NPSEs approximately 1 year after efavirenz has been initiated. This means that patient monitoring needs to be a continuous process. One study ${ }^{[25]}$ stated that the only side-effects that did resolve were the neurological side-effects as the psychiatric side-effects were not generally identified and addressed by physicians. It was suggested that the psychiatric status of patients initiated on efavirenz be closely 
monitored for at least the first 6 months to 1 year of treatment. ${ }^{[25]}$ Specific patients who should be monitored are those with early neurological side-effects, as well as those with a history of psychiatric disorders or substance abuse. For patients who are in hospital environments, daily mental status evaluations should be performed. For patients in outpatient settings, evaluations should be performed at every visit for at least 1 year after the initiation of efavirenz.

\section{Severity of NPSEs}

The severity of NPSEs may negatively influence the adherence of patients to efavirenz therapy. Studies generally show that the symptoms are mild and do not warrant the discontinuation of efavirenz. ${ }^{[10,14,22,28]}$ It has, however, been stated that if the side-effects persist, patient adherence may decline over time regardless of whether or not the NPSEs are mild to moderate in severity. ${ }^{[17,25,28]}$ Ongoing patient counselling and monitoring is thus imperative for patients using efavirenz, as it is for all patients on ART.

\section{Management of NPSEs}

The Clinical Guidelines for the Management of HIV and AIDS in Adults and Adolescents in South Africa ${ }^{[19]}$ do not discuss the management of NPSEs caused by efavirenz in depth. Studies ${ }^{[21,25]}$ show that efavirenz is discontinued in patients who are unable to tolerate the side-effects. It is not indicated whether or not another antiretroviral agent is substituted in its place or if efavirenz is reinstated once the side-effects have resolved. The Clinical Guidelines ${ }^{[19]}$ state that an acute psychotic episode should be managed according to the Standard Treatment Guidelines, ${ }^{[29]}$ which recommend that an acute psychotic episode be managed with 2 - $5 \mathrm{mg}$ of haloperidol administered intramuscularly, to be repeated in 60 minutes if required. If the response to haloperidol is poor, a benzodiazepine such as lorazepam may be administered intramuscularly at a dose of $1-4 \mathrm{mg} .^{[29]}$

The NPSEs caused by efavirenz should be treated according to severity. ${ }^{[30]}$ Mild symptoms can be monitored and treated pharmacologically if necessary, whereas more severe symptoms may warrant the discontinuation of efavirenz and the addition of an alternative antiretroviral agent. Patients with a history of depression should receive antidepressant therapy before the initiation of ART or concurrently. ${ }^{[31]}$ If depression occurs after the initiation of efavirenz and does not resolve spontaneously, alternative ART (as ART is always a combination of three agents) or aggressive antidepressant treatment may need to be considered. Depression may be treated with selective serotonin reuptake inhibitors such as citalopram, escitalopram or fluoxetine. ${ }^{[32,33]}$ If a patient experiences acute psychosis, antipsychotic agents are safe to use. ${ }^{[34]}$ These agents should always be used at the lowest possible dose for the shortest duration possible. Generally, typical antipsychotic agents such as haloperidol or chlorpromazine are used in resource-limited settings. The patients should be monitored for extrapyramidal symptoms. If risperidone, an atypical antipsychotic, is available, it may be used at a dose of 1 - 4 mg daily. ${ }^{[34]}$ Atypical antipsychotics are recommended over typical antipsychotics as they are better tolerated, but this does not mean that they are without side-effects. Atypical antipsychotics are associated with long-term metabolic effects and the potential for drug interactions. ${ }^{[34]}$ Patients who have been experiencing NPSEs with efavirenz for years may be treated symptomatically if necessary.
The patient may still be on efavirenz because the patient finds the side-effects tolerable. Indeed, one of the studies that demonstrated delayed onset or persistence of NPSEs indicates that the long-term effects may be mild and tolerated well by patients. ${ }^{[17]}$

Nevirapine may be substituted for efavirenz if the patient finds the NPSEs intolerable. Research has shown that this substitution will result in the resolution of NPSEs. ${ }^{[35]}$ However, there are safety concerns with nevirapine in terms of skin abnormalities and hepatitis, which is a life-threatening reaction. ${ }^{[35]}$ Patients being initiated on nevirapine need to be reviewed during the first 2 weeks, as recommended by the Guidelines. ${ }^{[4]}$ The other alternative as indicated by the Guidelines is lopinavir/ritonavir. However, there are significant drug interactions between ritonavir and psychotropic drugs such as clozapine, carbamazepine, and sedatives and hypnotics such as diazepam, midazolam and zolpidem. ${ }^{[36]}$ This would result in further problems in managing the psychiatric patient and the benefits should be weighed against the potential risks before initiating this agent. Other options would be the integrase-inhibitor raltegravir ${ }^{[36]}$ or the non-nucleoside reverse transcriptase inhibitor rilpivirine. ${ }^{[37]}$ Virological failure is more prevalent with rilpivirine than efavirenz, but the side-effect profile of rilpivirine is superior to efavirenz, specifically in terms of NPSEs. ${ }^{[37]}$

Currently, clinical practice does not favour the prescribing of efavirenz to patients with pre-existing psychiatric conditions, which may compromise the quality of virological control. It is interesting to note that the Guidelines state that efavirenz is contraindicated in patients with an active psychiatric illness and nevirapine or lopinavir/ritonavir should be considered instead. ${ }^{[4]}$ Considering that NPSEs of efavirenz are not generally severe enough to warrant the discontinuation of the medicine, there is cause for reconsideration of this matter. Improved compliance may be possible with use of the fixed-dose combination in patients with an active psychiatric illness in order to reduce an already extensive pill burden, as well as improved virological control.

Declaration. We declare that we have no financial or personal relationships which may have inappropriately influenced us in writing this paper.

\section{References}

1. Statistics South Africa. Mid-year population estimate. 2013. http://www.statssa.gov.za/ publications/P0302/P03022013.pdf (accessed 13 February 2015).

2. AIDS Foundation South Africa. http://www.aids.org.za/hivaids-in-south-africa/ (accessed 12 September 2013).

3. Avert: AVERTing HIV and AIDS. http://www.avert.org/hiv-and-aids-treatment-care.htm (accessed 13 February 2015).

4. National Consolidated Guidelines for the Prevention of Mother-to-child Transmission of HIV (PMTCT) and the Management of HIV in Children, Adolescents and Adults. 2014. Pretoria: National Department of Health, 2014.

5. Cavalcante GIT, Capistrano VLM, Cavalcante FSD, et al. Implications of efavirenz for neuropsychiatry: A review. Int J Neurosci 2010;120(12):739-745. [http://dx.doi.org/10.3109/ 00207454.2010.520541]

6. Piscitelli SC. Space out drug discontinuations prior to 'drug holiday'? Medscape. 2000. http:// www.medscape.com/viewarticle/413306 (accessed 1 September 2014).

7. Mukonzo JK, Okwera A, Nakasujja N, et al. Influence of efavirenz pharmacokinetics and pharmacogenetics on neuropsychological disorders in Ugandan HIV-positive patients with or without tuberculosis: A prospective cohort study. BMC Infect Dis 2013;13(261):1-11. [http:// dx.doi.org/10.1186/1471-2334-13-261]

8. Mukonzo JK, Owen JS, Ogwal-Okeng J, et al. Pharmacogenetic-based efavirenz dose modification: Suggestions for an African population and the different CYP2B6 genotypes. Plos One 2014;9(1):1-9. [http://dx.doi.org/10.1371/journal.pone.0086919]

9. Naidoo P, Chetty VV, Chetty M. Impact of CYP polymorphisms, ethnicity and sex differences in metabolism on dosing strategies: The case of efavirenz. Eur J Clin Pharmacol 2014;70(4):379-389. [http://dx.doi.org/10.1007/s00228-013-1634-1] 


\section{fre

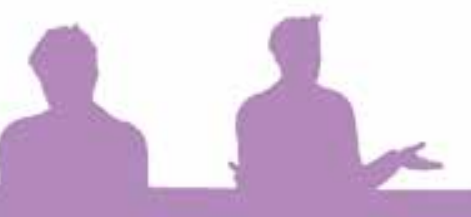 $\mathrm{Ar}$

10. Gutierrez-Valencia A, Viciana P, Palacios R, et al. Stepped-dose versus full dose efavirenz for HIV infection and neuropsychiatric adverse events: A randomized trial. Ann Intern Med 2011;151(3):149-156. [http://dx.doi.org/10.7326/0003-4819-151-3-200908040-00127]

11. Kenedi CA, Goforth HW. A systematic review of the psychiatric side-effects of efavirenz. AIDS Behav 2011;15(8):1803-1808. [http://dx.doi.org/10.1007/s10461-011-9939-5]

12. Lochet P, Peyriere H, Lotthe A, Mauboussin JM, Delmas B, Reynes J. Long-term assessment of neuropsychiatric adverse reactions associated with efavirenz. HIV Med 2003;4(1):62-66 [http://dx.doi.org/10.1046/j.1468-1293.2003.00136.x]

13. Arendt G, de Nocker D, von Giesen H, Nolting T. Neuropsychiatric side effects of efavirenz therapy. Expert Opin Drug Saf 2007;6(2):147-154. [http://dx.doi. org/10.1517/14740338.6.2.147]

14. Nelson M, Stellbrink H, Podzamczer D, et al. A comparison of neuropsychiatric adverse events during 12 weeks of treatment with etravirine and efavirenz in a treatment naive, HIV-1-infected population. AIDS 2011;25(3):335-340. [http://dx.doi.org/10.1097/ QAD.0b013e3283416873]

15. Journot V, Chene G, de Castro N, et al. Use of efavirenz is not associated with a higher risk of depressive disorders: A substudy of the randomized clinical trial ALIZE-ANRS 099. Clin Infect Dis 2006;42(12):1790-1799. [http://dx.doi.org/10.1086/504323]

16. Deeks SG, Lewin SR, Havlir DV. The end of AIDS: HIV infection as a chronic disease. Lancet 2013;382(9903):1525-1533. [http://dx.doi.org/10.1016/S0140-6736(13)61809-7]

17. Fumaz CR, Munoz-Moreno JA, Molto J, et al. Long-term neuropsychiatric disorders on efavirenz-based approaches: quality of life, psychological issues, and adherence. J Acq Immun Def Synd 2005;38(5):560-565. [http://dx.doi.org/10.1097/01.qai.0000147523.41993.47]

18. Clifford DB, Evans S, Yang Y, Acosta EP, Ribaudo H, Gulick RM. Long-term impact of efavirenz on neuropsychological performance and symptoms in HIV-infected individuals (ACTG5097s). HIV Clin Trials 2009;10(6):343-355.

19. Clinical Guidelines for the Management of HIV and AIDS in Adults and Adolescents. Pretoria: National Department of Health, 2010.

20. Adco-Efavirenz package insert. Midrand (Gauteng): Adcock Ingram, 2007.

21. Gazzard B, Balkin A, Hill A. Analysis of neuropsychiatric adverse events during clinical trials of efavirenz in antiretroviral-naïve patients: A systematic review. AIDS Rev 2010;12(2):67-75.

22. Marzolini C, Telenti A, Decosterd LA, Greub G, Biollaz J, Buclin T. Efavirenz plasma levels can predict treatment failure and central nervous system side effects in HIV-1 infected patients. AIDS 2001;15(1):71-75. [http://dx.doi.org/10.1097/00002030-200101050-00011]

23. Hawkins T, Geist C, Young B, et al. Comparison of neuropsychiatric side effects in an observational cohort of efavirenz- and protease inhibitor-treated patients. HIV Clin Trials 2005;6(4):187-196.
24. Gounden V, van Niekerk C, Snyman T, George JA. Presence of CYP2B6 516G> T polymorphism, increased plasma efavirenz concentrations and early neuropsychiatric side effects in South African HIV-infected patients. AIDS Res Ther 2010;7(32):1-9. [http://dx.doi. org/10.1186/1742-6405-7-32]

25. Lennox JL, DeJesus E, Lazzarin A, et al. Safety and efficacy of raltegravir-based versus efavirenz-based combination therapy in treatment-naïve patients with HIV-1 infection: A multicentre, double-blind, randomised controlled trial. Lancet 2009;374(9692):796-806. [http://dx.doi.org/10.1016/s0140-6736(09)60918-1]

26. Orkin C, Nelson M, Katlama C, et al. Changes in patient-reported neuropsychiatric outcomes during the SENSE trial: First-line treatment with two nucleoside analogues plus etravirine or efavirenz. Health Outcomes Res Med 2012;3(3):113-119. [http://dx.doi.org/10.1016/j. ehrm.2012.06.002]

27. Gutierrez F, Navarro A, Padillo S, et al. Prediction of neuropsychiatric adverse events associated with long-term efavirenz therapy, using plasma drug level monitoring. Clin Infect Dis 2005;41(11):1648-1653. [http://dx.doi.org/10.1086/497835]

28. Rihs TA, Begley K, Smith DE, et al. Efavirenz and chronic neuropsychiatric symptoms: A cross-sectional case control study. HIV Med 2006;7(8):544-548. [http://dx.doi.org/10.1111/ j.1468-1293.2006.00419.x]

29. Standard Treatment Guidelines and Essential Medicines List for South Africa: Hospital Level Adults. Pretoria: National Department of Health, 2012.

30. Turjanski N, Llyod GG. Psychiatric side effects of medications: Recent developments. Adv Psychiatr Treat 2005;11:58-70. [http://dx.doi.org/10.1192/apt.11.1.58]

31. Treisman GJ, Kaplin AI. Neurologic and psychiatric complications of antiretroviral agents. AIDS 2002;16(9):1201-1215. [http://dx.doi.org/10.1097/00002030-200206140-00002]

32. Brogan K, Lux J. Management of common psychiatric conditions in the HIV-positive population. Curr HIV/AIDS Rep 2009;6(2):108-115. [http://dx.doi.org/10.1007/s11904-0090016-x]

33. Ferrando SJ. Psychopharmacologic treatment of patients with HIV/AIDS. Curr Psychiatry Rep 2009;11(3):235-242. [http://dx.doi.org/10.1007/s11920-009-0036-7]

34. Jonsson G, Joska JA. Assessment and treatment of psychosis in people living with HIV/AIDS. S Afr J HIV Med 2009;10(3):20-27.

35. Schouten JT, Krambrink A, Ribaudo HJ, et al. Substitution of nevirapine because of efavirenz toxicity in AIDS Clinical Trials Group A5095. Clin Infect Dis 2010;50(5):787-791. [http:// dx.doi.org/10.1086/650539]

36. Rossiter D, ed. South African Medicines Formulary. 10th ed. Cape Town: Health and Medical Publishing Group, 2012.

37. Claydon P, ed. HIV Treatment Bulletin + South. HIV i-Base 2012;5(1):1-42. 